\title{
Direct measurement of refracted trajectory of transmitting electron cyclotron beam through plasma on the Large Helical Device
}

\author{
Hiromi Takahashi ${ }^{1, a}$, Shin Kubo ${ }^{1}$, Takashi Shimozuma ${ }^{1}$, Hiroe Igami ${ }^{1}$, Yasuo Yoshimura ${ }^{1}$, Satoshi Ito ${ }^{1}$, \\ Sakuji Kobayasi ${ }^{1}$, Yoshinori Mizuno ${ }^{1}$, Kohta Okada ${ }^{1}$, Shuji Kamio ${ }^{1}$, Takashi Mutoh ${ }^{1}$, Masaki Osakabe ${ }^{1}$, \\ Kazunobu Nagasaki ${ }^{2}$, Nikolai B. Marushchenko ${ }^{3}$, Yuriy Turkin ${ }^{3}$, and the LHD Experiment Group ${ }^{1}$ \\ ${ }^{1}$ National Institute for Fusion Science, Toki 509-5292, Japan \\ ${ }^{2}$ Institute of Advanced Energy, Kyoto Univ., Uji 611-0011, Japan \\ ${ }^{3}$ Max-Planck-Institut für Plasmaphysik, EURATOM Association, D-17491 Greifswald, Germany
}

\begin{abstract}
The electron-cyclotron (EC) -beam refraction due to the presence of plasma was investigated in the Large Helical Device. The transmitted-EC-beam measurement system was constructed and the beam pattern on the opposite side of the irradiated surface was measured using an IR camera. Clear dependence of the EC-beam refraction on the electron density was observed and the beam shift in the toroidal direction showed good agreement with the ray-trace calculation of TRAVIS. The influence of the peripheral density profile and the thermal effect on the beam refraction were discussed.
\end{abstract}

\section{Introduction}

In toroidal devices, the importance of local and effective electron heating with an electron cyclotron (EC) beam has been widely recognized [1,2]. Electron cyclotron resonance heating $(\mathrm{ECRH})$ has been used for the control of local plasma parameters such as a radial electric field, particle/heat flux [3], and toroidal current [4-6], and for stabilization of neoclassical tearing mode [7-9]. ECRH is also expected as an essential heating and a current drive source in advanced toroidal devices such as ITER [10, $11]$ and Wendelstein $7-X[12,13]$. In order to ensure the effective heating and the controllability of local-plasma parameters by ECRH, investigation of the EC-beam refraction is important. Although the refraction of an EC beam in the electron density $\left(n_{\mathrm{e}}\right)$ gradient and/or near the EC resonance layer, which is related to the thermal effect, has been predicted [14-16], that has not been verified precisely by experiments up to now.

A target plate facing on the ECRH antenna has been set inside the vacuum vessel on the Large Helical Device (LHD) in order to measure the transmitted power of EC beam, which is not absorbed into the plasma in the single path. The LHD $[17,18]$ is a suitable machine for direct measurement of transmitted-EC beam and for the validation of the EC-beam refraction because the LHD has many ports for installing the beam target and is equipped with the high-power ECRH system [19-21].

This paper shows the observation results of EC beam refraction due to the presence of the plasma in the LHD and is organized as follows. Section 2 deals with the
transmitted-EC-beam measurement. The set-up of the EC-beam target plate inside the LHD and the observation of the EC beam refraction due to the existence of plasmas are shown. Section 3 is the discussion and the experimental-EC-beam refraction is compared with the ray-trace results under several calculation conditions in order to investigate the refraction effect due to the density-gradient and the anomalous dispersion (thermal effect). The summary is in section 4 .

\section{Transmitted EC beam measurement}

\subsection{Set-up of target plate in the LHD}

Figure 1 shows (a) the transmitted-EC-beammeasurement system in the LHD and (b) the enlarged illustration. The EC-beam-target plate (diameter: $280 \mathrm{~mm}$, thickness: $15 \mathrm{~mm}$ ) made of isotropic graphite has been set $2.5 \mathrm{~m}$ lower from the equatorial plane of the LHD. The center position of the target plate is $R=3.59 \mathrm{~m}, Z=$ $-2.5 \mathrm{~m}$, and $t=0 \mathrm{~m}$, where $R, Z$, and $t$ are the radial, vertical and toroidal directions, respectively. The system consists of the target plate, a SUS cylinder, an Al mirror, a $\mathrm{BaF}_{2}$ window and an IR camera. The temperature rise of the plate due to the EC beam has been measured using the IR camera. The SUS cylinder (inner diameter: 256 $\mathrm{mm}$, thickness: $2 \mathrm{~mm}$ ) is used to protect the $\mathrm{BaF}_{2}$ window and the IR camera from the stray EC wave. The camera is covered using a shield box (thickness: $14 \mathrm{~mm}$ ) made of $\mathrm{PB}$ permalloy, which is the alloyed metal of $\mathrm{Ni}$ and $\mathrm{Fe}$, to

\footnotetext{
${ }^{\mathrm{a}}$ Corresponding author: takahashi.hiromi@LHD.nifs.ac.jp
} 

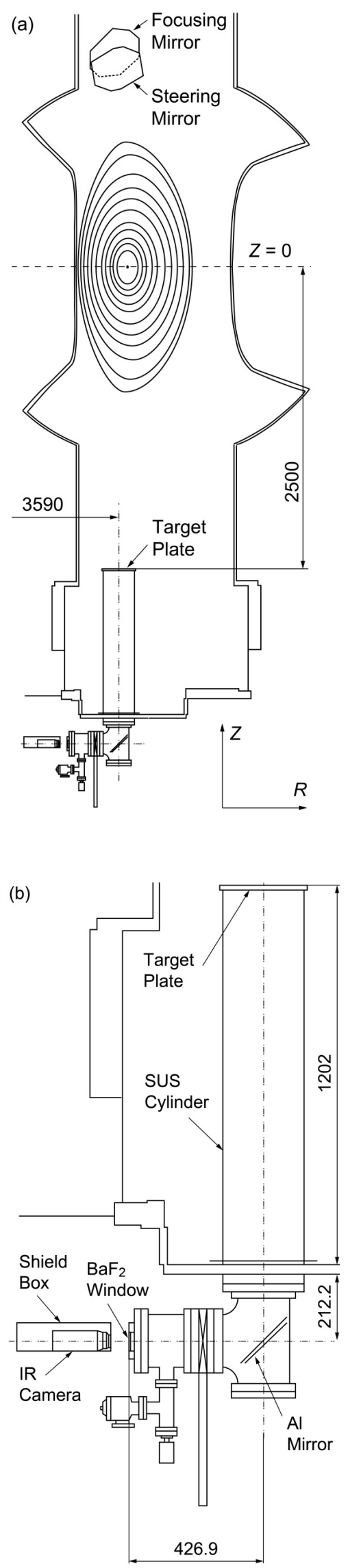

Figure 1. (a) The transmitted-EC-beam-measurement system in the LHD and (b) the enlarged illustration. reduce the effect of stray magnetic field from the LHD on the IR camera. The target plate receives the EC beam ejected from the facing-on upper launcher.

Figure 2 shows (a) the target plate and the expected beam cross-section defined by the electric field $e$-holding size of the injected Gaussian beam on the $R-t$ plane, the radial profiles of (b) the local deposition power $d P_{\text {dep }} / d V$ and (c) the volume integral of $d P_{\text {dep }} / d V$ normalized by the injection power $P$. The words $\mathrm{CW}$ and $\mathrm{CCW}$ in Fig. 2 (a) represent toroidal clockwise and toroidal-counter clockwise, respectively. In the label of horizontal axis in Figs. 2 (b) and (c), $r_{\text {eff }}$ is the effective minor radius and $a_{99}$ is the averaged minor radius in which $99 \%$ of the stored energy is confined. The deposition power was calculated for the second-harmonic-ordinary $(\mathrm{O} 2)$ mode using TRAVIS code [22] with the weakly-relativisticdielectric tensor under the same experimental condition with that of the line-averaged electron density of $0.4 \times 10^{19}$ $\mathrm{m}^{-3}$, which is shown later in Figs. 3 (c) and (d). The EC beam treated in the TRAVIS code consists of 25 rays. The antenna used in the transmitted EC beam experiments is set at the upper port of the LHD and the center position of the final mirror (the steering mirror shown in Fig. 1 (a)) is $R=3.585 \mathrm{~m}, Z=1.6235 \mathrm{~m}$, and $t=$ $-0.2043 \mathrm{~m}$. The broken lines represent the cross-section of the injected beam when the EC beam is focused on $R=$ $3.6 \mathrm{~m}, Z=0 \mathrm{~m}$, and $t=0 \mathrm{~m}$, and the antenna setting is frequently used for the usual LHD experiments. The solid lines are those focusing on the center of the target plate and most parts of the EC beam lands on the target plate when the beam is not refracted. In the case EC beam is focused on the target plate, there is $0.14 \mathrm{~m}$ difference in the equatorial plane of the LHD in the $\mathrm{CW}$ direction from the usual operation, which corresponds to $\sim 1.5$ degrees of the toroidal angle. Although the peak position of the local deposition power slightly shifts outward, there is no clear difference in the total absorption power into the plasma compared with those in the normal operation.

\subsection{Experimental results}

In order to verify the dependence of the beam refraction on the plasma parameters, three conditions were selected for the experiments, such as no plasma, the line-averaged electron density of 0.2 , and $0.4 \times 10^{19} \mathrm{~m}^{-3}$. In the experiments, the $77-\mathrm{GHz}$ EC beam with $560 \mathrm{~kW} / 0.3 \mathrm{~s}$ was superposed on the plasma sustained by an NBI as the $\mathrm{O} 2$ mode under the magnetic configuration with the magnetic axis position of $3.6 \mathrm{~m}$ and the magnetic field of $1.375 \mathrm{~T}$, where the EC beam absorption to the plasma was expected to be considerably small. Figure 3 shows the radial profiles of (a) the electron temperature $T_{\mathrm{e}}$, (b) the electron density $n_{\mathrm{e}}$, (c) $d P_{\mathrm{dep}} / d V$, and (d) $P_{\mathrm{dep}} / P$ in the target plasmas. In the case of $n_{\mathrm{e}}=0.4 \times 10^{19} \mathrm{~m}^{-3}$, steeper density gradient was formed in the plasma edge and the absorption power was 1.7 times larger compared with those of $n_{\mathrm{e}}=0.2 \times 10^{19} \mathrm{~m}^{-3}$. On the other hand, the $T_{\mathrm{e}}$ profiles were similar between these two conditions.

Figure 4 shows the profiles of the temperature rise of the target plate in three experimental conditions. The circles each indicated by the broken line represent the 

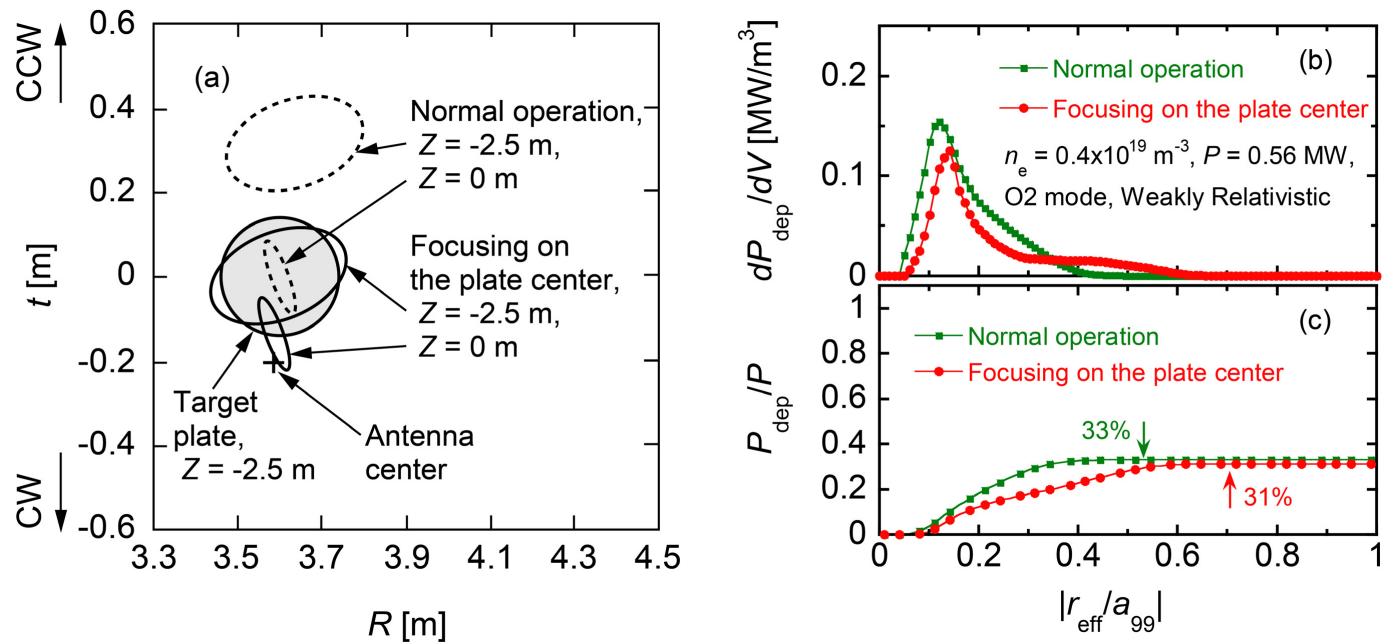

Figure 2. (a) The target plate and the expected beam cross-section on the $R$ - $t$ plane, the radial profiles of (b) $d P_{\text {dep }} / d V$, and (c) the volume integral of $d P_{\mathrm{dep}} / d V$ normalized by $P$.
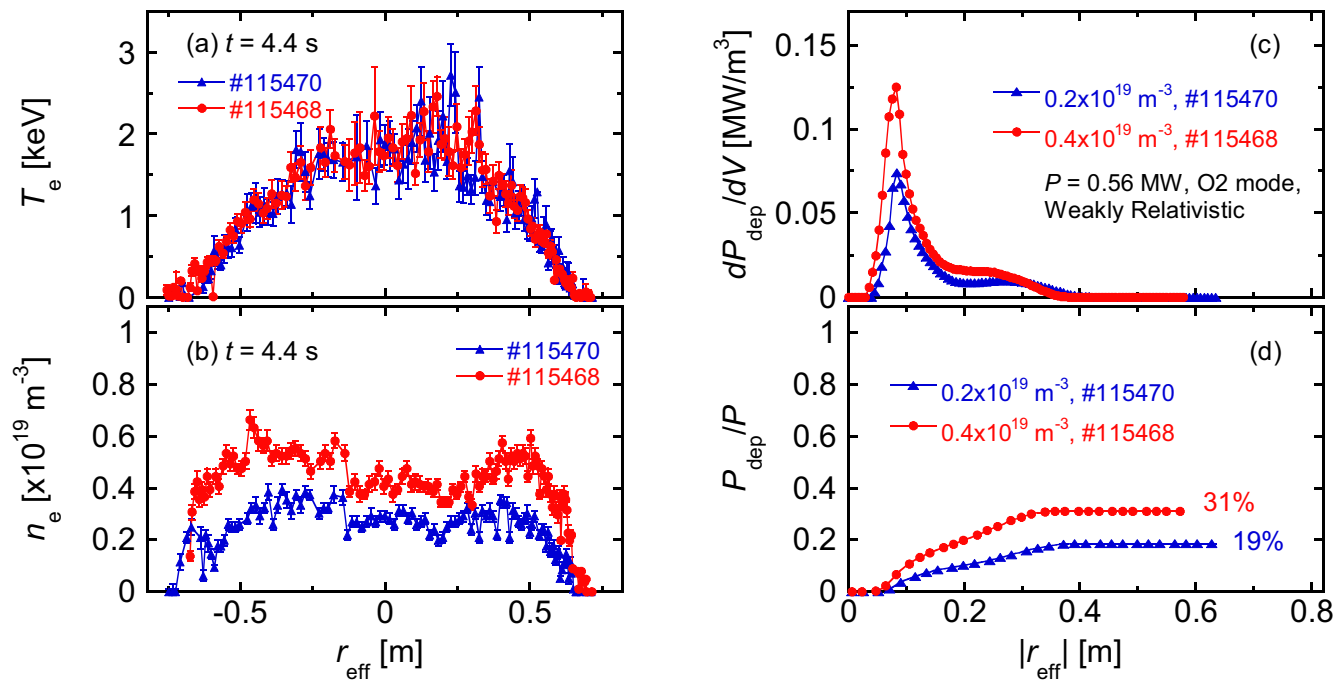

Figure 3. The radial profiles of (a) $T_{\mathrm{e}}$, (b) $n_{\mathrm{e}}$, (c) $d P_{\mathrm{dep}} / d V$, and (d) $P_{\mathrm{dep}} / P$ in the target plasmas.

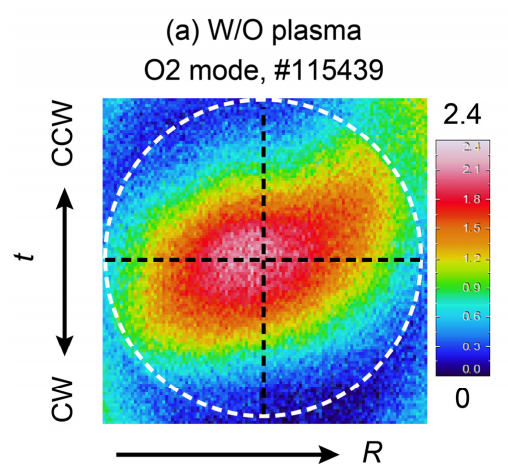

(b) $n_{\mathrm{e}} \sim 0.2 \times 10^{19} \mathrm{~m}^{-3}$

O2 mode, \#115470

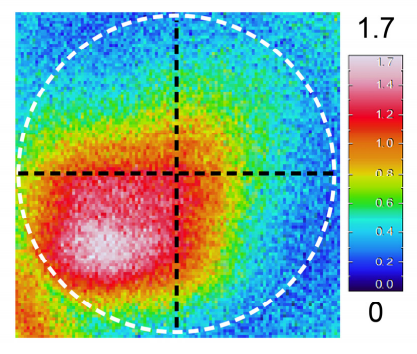

(c) $n_{\mathrm{e}} \sim 0.4 \times 10^{19} \mathrm{~m}^{-3}$

O2 mode, \#115468

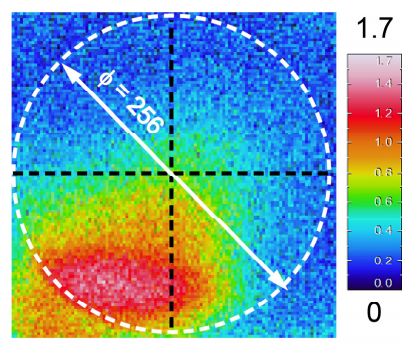

Figure 4. The profiles of the temperature rise of the target plate in three experimental conditions.

measurable boundary of the target plate. The EC beam was focused on the center of the target plate in all cases. Clear dependence of the beam refraction on $n_{\mathrm{e}}$ and/or its gradient was observed from the landing-point of the EC beam on the target plate. In the no plasma case, there is no effect of beam refraction thus the highest temperature rise was observed around the center of the target plate and the profile of the temperature rise was similar with that of the EC-beam-power density. On the other hand, the beam landing point moved in the $-R$ direction in the case with the plasma and the displacement in the $\mathrm{CW}$ direction became larger with increase in $n_{\mathrm{e}}$ and/or its shear. The peak-temperature rise became low due to the presence of plasmas. This is explained from that the EC 
beam was partially absorbed into the plasma and the power of the transmitted EC beam became small. Also the peak temperature rise in $n_{\mathrm{e}}=0.4 \times 10^{19} \mathrm{~m}^{-3}$ case was lower than that in $n_{\mathrm{e}}=0.2 \times 10^{19} \mathrm{~m}^{-3}$ because of the larger absorption power into the plasma, and was consistent with the ray-trace calculation result shown in Fig. 3 (c).

\section{Discussions}

Here the effect of the $n_{\mathrm{e}}$ profile in the peripheral region and the dielectric tensor (thermal effect) on the EC-beam refraction is investigated using TRAVIS code. Figure 5 shows the radial profiles of $T_{\mathrm{e}}$ and $n_{\mathrm{e}}$ used in the calculation. (a) and (b) are for the case of the lineaveraged-electron density of $0.2 \times 10^{19} \mathrm{~m}^{-3}$, and (c) and (d) are for $0.4 \times 10^{19} \mathrm{~m}^{-3}$. Two kinds of $n_{\mathrm{e}}$ profiles were prepared. Ones indicated by the solid lines were fitting data with the experimental $n_{\mathrm{e}}$ profiles in the whole plasma region. And the ones indicated by the broken lines were similar with the solid one, however these were assumed to be zero in the peripheral region. In usual LHD experiments, $n_{\mathrm{e}}$ profiles have long decay length in the plasma boundary and the finite value of $n_{\mathrm{e}}$ remains at the last-closed-flux surface (LCFS). Note that the equilibrium file, of which data exist only inside the LCFS, is used in the present calculation of TRAVIS for the LHD and $n_{\mathrm{e}}$ outside of LCFS is treated as zero. Therefore, the discontinuity of $n_{\mathrm{e}}$ profile occurred at the plasma boundary when the experimental fitting data is applied to the calculation. On the other hand, continuous $n_{\mathrm{e}}$ profile can be provided when $n_{\mathrm{e}}$ is forced to be zero in the plasma edge, although the profile is not matched with the experimental data in the peripheral region.

Figure 6 shows the trajectory of the EC-beam center on the $R-Z, t-Z$, and $R-t$ plane for (a)-(c) $n_{\mathrm{e}}=0.2 \times 10^{19} \mathrm{~m}^{-3}$ and (d)-(f) $0.4 \times 10^{19} \mathrm{~m}^{-3}$. Four curves illustrated in Figs. 6 (a)-(f) are the calculation results with the combination of the dielectric tensor of cold/weakly relativistic and the $n_{\mathrm{e}}$ near the plasma boundary of zero/finite value. The positions of the LCFS are shown by the broken lines in Figs. 6 (a), (b), (d), and (e). The circled points in Figs. 6 (c) and (f) are the landing points of the EC beam center on the target plate. In the case of $n_{\mathrm{e}}=0.2 \times 10^{19} \mathrm{~m}^{-3}$, the EC-beam refraction in the radial direction depended on the shape of $n_{\mathrm{e}}$ profile, namely, the displacement became large for the profiles with $n_{\mathrm{e}}(1.0) \sim 0$. While the significant difference of the beam displacement depending on the calculation conditions was not shown in the toroidal direction and the displacement was $\sim 0.03 \mathrm{~m}$ in the $\mathrm{CW}$ direction at $Z=-2.5 \mathrm{~m}$. In the case of $n_{\mathrm{e}}=$ $0.4 \times 10^{19} \mathrm{~m}^{-3}$, clear dependence of the beam trajectory both on $n_{\mathrm{e}}$ profile and the dielectric tensor was found in the radial direction. The magnitude of the refraction became larger in the case using $n_{\mathrm{e}}$ profiles with $n_{\mathrm{e}}(1.0) \sim 0$ and the weakly-relativistic dielectric tensor. Also the ECbeam refraction was affected due to the difference in the edge $n_{\mathrm{e}}$ profile rather than that in the dielectric tensor. The displacement in the toroidal-CW direction was shown in all conditions and was $\sim 0.008 \mathrm{~m}$ larger in the cases of $n_{\mathrm{e}}$ profile with $n_{\mathrm{e}}(1.0) \sim 0$ than those with finite edge $n_{\mathrm{e}}$.

Figure 7 shows the radial profiles of $n_{\mathrm{e}}$ with (a) $n_{\mathrm{e}}(1.0) \sim 0$, (b) the finite edge value, the dependence of $\Delta R$ and $\Delta t$ on $n_{\mathrm{e}}$ with the dielectric tensor and the edge $n_{\mathrm{e}}$ for the calculation of (c) cold and $n_{\mathrm{e}}(1.0) \sim 0$, (d) cold and finite, (e) weakly relativistic and $n_{\mathrm{e}}(1.0) \sim 0$ and (f) weakly relativistic and finite. Here $\Delta R$ and $\Delta t$ represent the displacement in the radial and the toroidal direction, respectively. The solid and the broken lines represent the experimental results and the calculation of TRAVIS, respectively. The calculation in the toroidal direction showed good agreement with the experiment in all cases and little difference existed among them. While in the radial direction, the displacement was smaller in the calculation than those in the experiment. Although the calculated $\Delta R$ shown in Fig. 7 (e) were the closest to the experimental values in the four cases, the polarity of $d \Delta R / d n_{\mathrm{e}}$ was negative in $0.2<n_{\mathrm{e}}<0.4$ and the tendency was different from that in the experiment. A ray-trace calculation with appropriate experimental conditions, such as an electron density profile and magnetic field structure in the plasma peripheral region, a proper EC beam profile and a dielectric tensor, is necessary in order to explain the experimental results. The peripheral magnetic structure and rather long density decay length extending over confinement region affect ray trajectory and polarization (ordinary- and extraordinary-mode (X mode) content) as well.

The difference in the magnitude of the EC-beam refraction observed in the experiments is considered mainly due to the effect of $n_{\mathrm{e}}$ and/or its shear, not the $T_{\mathrm{e}}$ effect because $T_{\mathrm{e}}$ profile is almost the same between the two experimental conditions as shown in Fig. 3 (a). ECbeam-transmitted experiments with wider temperature regime and survey of incident positions of EC beam are necessary for the verification of the thermal effect, in other words, the effect by the electron temperature and by an angle between EC beam and the resonance surface. The beam polarization scanning is a possible scenario to be taken in order to clarify the thermal effect in the beam refraction. It is noted in theory that the thermal effect in the beam refraction strongly depends on the beam polarization, namely, the resonant interaction on the thermal effect is scaled $\left(T_{\mathrm{e}} / m c^{2}\right)^{2}$ for the $\mathrm{O} 2$ mode, which was treated in the present study, and is of order $T_{\mathrm{e}} / m c^{2}$ for the fundamental $\mathrm{O}$ mode and the X2 mode [14]. Also, detailed investigation of the refraction dependence on $n_{\mathrm{e}}$ must be carried out. In the system introduced here, the landing point of EC-beam was close to the boundary of the measurable region in the target plate even in low $n_{\mathrm{e}}$ of $0.4 \times 10^{19} \mathrm{~m}^{-3}$ as shown in Fig. 4 (c). In order to detect the refracted-transmitted beam in wide-plasma-parameter range and several-EC-beam condition, system upgrade, such as the installation of the larger target plate and the setting of the plate closer to plasma, is necessary. Recently, the preliminary data began to be obtained using a new system [23] and the detailed analysis results will be shown in the near future. 

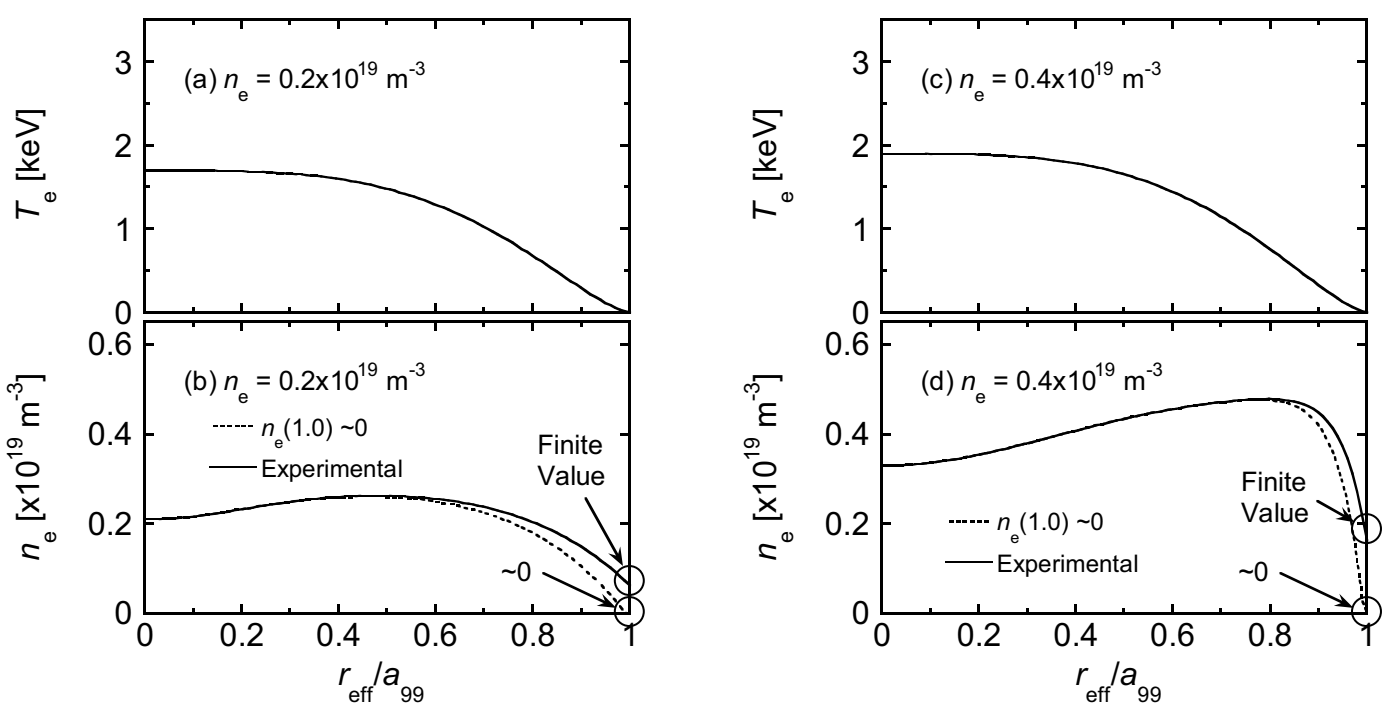

Figure 5. The radial profiles of $T_{\mathrm{e}}$ and $n_{\mathrm{e}}$ used in the calculation. (a) and (b) are for the case of the line-averaged-electron density of $0.2 \times 10^{19} \mathrm{~m}^{-3}$, and (c) and (d) are for $0.4 \times 10^{19} \mathrm{~m}^{-3}$.
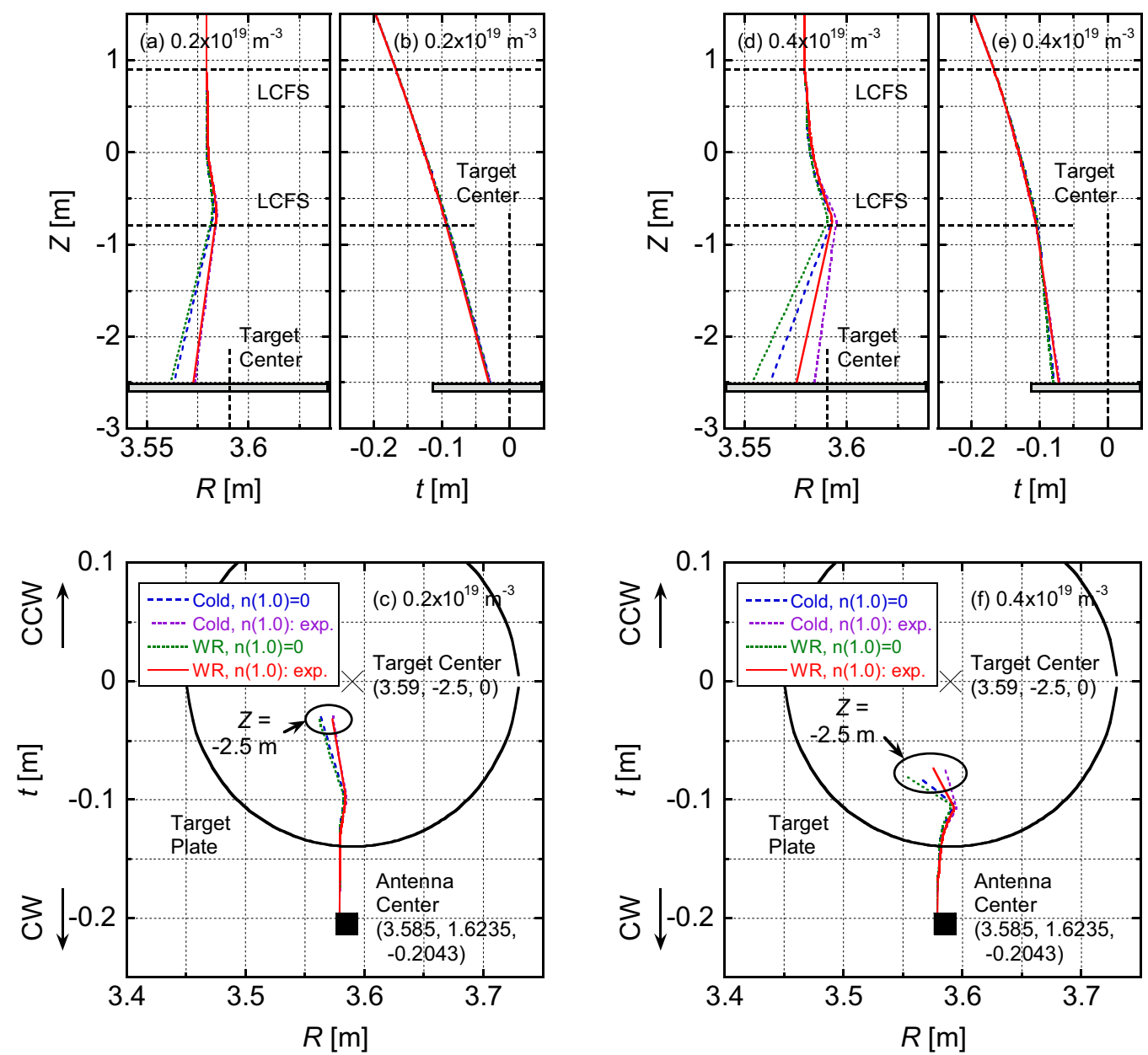

Figure 6. The trajectory of the EC-beam center on the $R-Z, t-Z$ and $R-t$ plane for (a)-(c) $n_{\mathrm{e}}=0.2 \times 10^{19} \mathrm{~m}^{-3}$ and (d)-(f) $0.4 \times 10^{19} \mathrm{~m}^{-3}$.

\section{Summary}

The EC-beam refraction due to the presence of the plasma was verified experimentally using the target plate inside the LHD. Three conditions were selected for the experiments such as no plasma, $n_{\mathrm{e}}=0.2$, and $0.4 \times 10^{19}$ $\mathrm{m}^{-3}$. In the experiments, the $77-\mathrm{GHz} \mathrm{EC}$ beam was injected as the second-harmonic-O mode intentionally in order to increase (decrease) the transmitted (absorbed) power. Clear dependence of the EC-beam refraction on $n_{\mathrm{e}}$ 


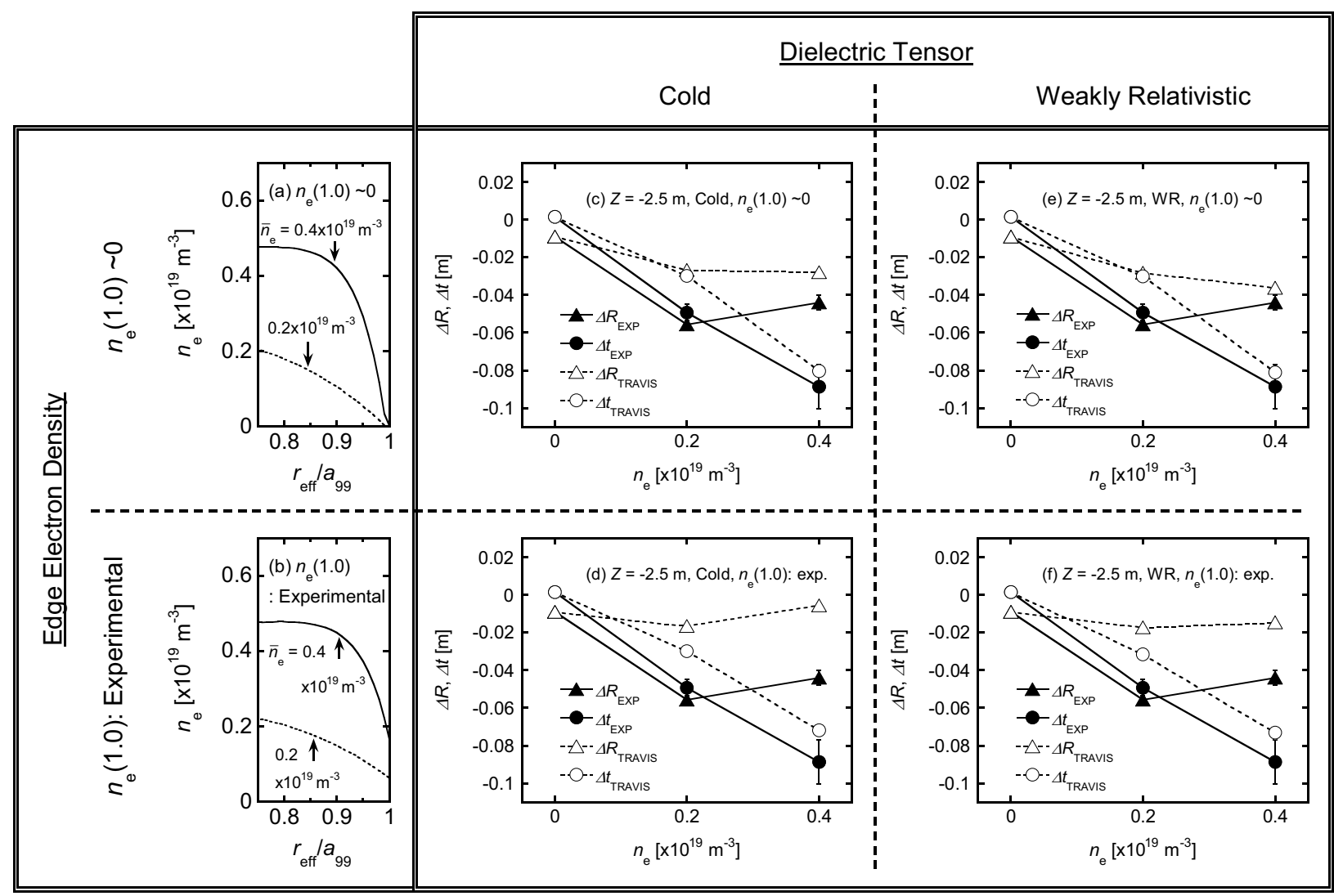

Figure 7. The radial profiles of $n_{\mathrm{e}}$ with (a) $n_{\mathrm{e}}(1.0) \sim 0$, (b) the finite edge value, the dependence of $\Delta R$ and $\Delta t$ on $n_{\mathrm{e}}$ with the dielectric tensor and the edge $n_{\mathrm{e}}$ for the calculation of (c) cold and $n_{\mathrm{e}}(1.0) \sim 0$, (d) cold and finite, (e) weakly relativistic and $n_{\mathrm{e}}(1.0) \sim 0$, and (f) weakly relativistic and finite.

was observed. The displacement of the beam center showed good agreement with the ray-trace calculation in the toroidal direction. On the other hand, the calculated $\Delta R$ was considerably smaller than the experimental value and the tendency on $n_{\mathrm{e}}$ changed both qualitatively and quantitatively due to the applied $n_{\mathrm{e}}$ profile and the dielectric tensor. A ray-trace calculation with appropriate experimental conditions is necessary in order to explain the experimental results. The experiments with wider plasma parameter regime and with several-incident conditions of EC-beam are necessary for deepening of an understanding of physical mechanism of EC-beam refraction. Those experimental results with upgraded system will be shown in the near future.

\section{Acknowledgments}

This work was supported by NIFS grants ULRR701 and 804.

\section{References}

1. V. Erckmann and U. Gasparino, Plasma Phys. Control. Fusion 361869 (1994)

2. B. Lloyd, Plasma Phys. Control. Fusion 40, A119 (1998)

3. H. Idei et al., Phys. Rev. Lett. 71, 2220 (1993)

4. H. Maassberg et al., Plasma Phys. Control. Fusion 47, 1137 (2005)
5. K. Nagasaki et al., Nucl. Fusion 50, 025003 (2010)

6. Y. Yoshimura et al., Fusion Sci. Technol. 58, 551 (2010)

7. K. Nagasaki et al., Nucl. Fusion 45, 1608 (2005)

8. R. Prater et al., Nucl. Fusion 47, 371 (2007)

9. A. Isayama et al., Nucl. Fusion 49, 055006 (2009)

10. T. Imai, Fusion Eng. Des. 55, 281 (2001)

11. F. Wagner, Plasma Phys. Control. Fusion 52, 124044 (2010)

12. V. Erckmann et al., Fusion Sci. Technol. 52, 291 (2007)

13. G. Gantenbein et al., J Infrared Milli Terahz Waves 32, 320 (2011)

14. E. Westerhof, Plasma Phys. Control. Fusion 39, 1015 (1997)

15. D. C. McDonald et al., Phys. Plasmas 5, 883 (1998)

16. M. D. Tokman, E. Westerhof and M.A. Gavrilova, Plasma Phys. Control. Fusion 42, 91 (2000)

17. H. Yamada for the LHD Experiment Group, Nucl. Fusion 51, 094021 (2011)

18. O. Kaneko et al., Nucl. Fusion 53, 104015 (2013)

19. T. Shimozuma et al., Fusion Sci. Technol. 58, 530 (2010)

20. H. Takahashi et al., Nucl. Fusion 53, 073034 (2013)

21. H. Takahashi et al., Phys. Plasmas 21, 061506 (2014)

22. N. B. Marushchenko et al., Plasma Fusion Research 2, S1129 (2007)

23. S. Kamio et al., submitted to Rev. Sci. Instrum. 